\title{
Save and Transmit Scheme for Energy Harvesting MIMO Systems with TAS/MRC
}

\author{
Issa Chihaoui and Mohamed Lassaad Ammari
}

\begin{abstract}
In this paper, we propose and analyze a wireless transmitter, for multiple-input multiple-output (MIMO) systems, that relies exclusively on energy harvesting. We consider wireless transceivers where the transmitter harvests the total required energy from its environment through various sources. We assume that both transmitter and receiver are equipped with multiple antennas. At the transmitter, a single transmit antenna that maximizes the signal-to-noise ratio (SNR) at the receiver is selected for transmission. The remaining antennas are used for energy harvesting. At the receiver side, maximal-ratio combining (MRC) is used. Furthermore, we assume that all the harvested power is used to power the transmitter immediately. The performance of the proposed scheme is analyzed in terms of outage probability (OP), symbol error rate (SER) and channel capacity. The harvested energy comes from random sources and is considered as a random variable. Assuming that the harvested power follows a gamma distribution and the MIMO channel is a Rayleigh flat fading process, we derive a closedform expressions for the exact cumulative distribution function (CDF) and probability density function (PDF) of the SNR. Based on this, we analyze the performance of the proposed energy harvesting scheme. The main contribution of this work consists on proposing a new energy harvesting protocol and providing an analytical performance assessment of this protocol over Rayleigh flat fading channels. The obtained analytical results are validated by comparing them with the results of Monte-Carlo (MC) simulations.
\end{abstract}

Index Terms-Energy harvesting, MIMO channels, TAS/MRC.

\section{INTRODUCTION}

Wireless communications with energy harvesting (EH) transmitters and/or receivers have recently attracted a considerable interest as a promising solution to design Self-Sustainable Standalone networks [1]-[7]. For these systems, the transmitter and/or the receiver scavenge energy from ambient power sources to power totally or partially the data transfer. In such a scenario, the harvested energy is not a deterministic quantity but a random process which varies in time at a scale on the order of the symbol duration [4]. In addition, the wireless communication channel has a stochastic fading. Consequently, the signal-to-noise ratio (SNR) at the receiver depends on two random variables (harvested energy and channel fading).

Manuscript received August 02, 2018; revised January 21, 2019. Date of publication February 28, 2019. The associate editor Prof. Zoran Blažević has been coordinating the review of this manuscript and approved it for publication.

I. Chihaoui is with the Innov'COM Laboratory, Higher School of Communication of Tunis, University of Carthage, Tunis, Tunisia. M. Lassaad Ammari is with the NOCCS Laboratory, National Engineering School of Sousse, University of Sousse, Tunisia. (emails: issa.chihaoui@mes.rnu.tn, mlammari@gel.ulaval.ca).

Digital Object Identifier (DOI): 10.24138/jcomss.v15i1.594
Obviously, the performance of EH systems depends on many other parameters such as circuit power consumption or battery efficiency. Hence, new transmission strategies are required to take advantage of the potentiality offered by the EH systems.

The EH process can be performed in either the transmit (TX) side or the receive (RX) side [7]. In this paper, we consider point-to-point multiple-input multiple-output (MIMO) communication systems using EH at the TX. For the EH TX scenario, we assume that the transmitter does not have a fixed power supply and needs to harvest energy from its environment through various different sources. Wireless systems with EH TX have be studied widely in the literature [1]-[10]. These works have focused on the design of energy scheduling policies for single-input single-output (SISO) systems using natural ambient energy sources. The proposed approaches can be classified into two main categories called offline and online polices [5]-[10]. The offline polices consider that channel state information (CSI) and energy state information (ESI) are available non-causally at the beginning of transmission [1]-[5] The online approaches assume a more realistic scenario that suggests the ESI are available causally [8]-[12]. For the offline energy management scheme, the problems of throughput maximization [1] and completion time minimization [2] have been investigated. The proposed solutions do not take into account the uncertainty of the amounts of the harvested energy. To overcome this limitation, online solutions for stochastic ESI model have been proposed [8]-[11]. For the online schemes, the problem of throughput maximization is considered and solved using the first-order Markov process [8]-[10]. In [11], authors have focused on EH systems with imperfect CSI. The design of wireless transmission systems with a TX relying exclusively on EH is proposed in [13]. Author in [14] has considered the energy harvesting for device-to-device communications in Nakagami fading channels with and decode-andforward (DF) scheme. Authors in [13] have presented a savethen-transmit (ST) protocol for SISO systems. In the proposed ST scheme, a fraction of the time is devoted exclusively for energy harvesting and the remaining fraction is used for data transmission. The first completely integrable circuits and architectures for RF energy harvesting systems are presented in [15].

This work proposes and analyzes a new energy harvesting scheme for (MIMO) systems. In fact, this paper considers an EH TX for MIMO systems. As in [13], we assume that the TX is exclusively powered by harvested energy. In [13], the proposed ST protocol assumed a time switching approach. To ensure transmission time continuity, we propose to replace 
time switching by the classical transmit antenna selection (TAS) scheme [16]. Hence, only a single transmit antenna, which maximizes the SNR at the receiver, is used for data transmission. The remaining transmit antennas are used for energy harvesting. Furthermore, we assume that all the receive antennas are combined with the maximal-ratio combining (MRC) technique. These choices are made to minimize the system complexity and to maximize the amount of the harvested energy. To simplify the system performance analysis, we assume that the harvested energy is gamma distributed and the channel is modeled as a flat fading Rayleigh process. Based on these assumptions, we derive the probability density function (PDF) and the cumulative distribution function (CDF) of the SNR. Then, the performances of the propose EH TX scheme are analyzed in terms of outage probability (OP), symbol error rate (SER) and channel capacity. Closed-form expressions for these metrics are derived and compared with Monte-Carlo simulation results.

The remainder of this paper is organized as follows. In Section II, we introduce the system model and the EH TX protocol. Section III provides the exact expression of the CDF the SNR and the OP. The PDF of the SNR is given in Section IV, where we derive the channel capacity expression. Section VI analyses the performance of our protocol. It includes numerical results and provides the performance comparison between analysis and Monte Carlo simulation curves. Finally, a conclusion to this work is provided in Section VII.

\section{System Model ANd EH PRotocol}

In this paper, we consider an EH TX system over MIMO channels. We assume that the transmitter and the receiver are equipped with $N_{t}$ and $N_{r}$ antennas, respectively. At any transmission period, only one transmit antenna is selected for transmission and the other transmit antennas are used to harvest energy from the environment. At the receiver side, the $N_{r}$ receive antennas are used for MRC. We consider a Rayleigh block flat fading MIMO channel, where the fading coefficients remain fixed during a transmission block but vary from block to block [13]. The channel matrix is denoted by $\mathbf{H}=\left[\mathbf{h}_{1}, \cdots, \mathbf{h}_{N_{t}}\right] \in \mathbb{C}^{N_{r} \times N_{t}}$, where $\mathbf{h}_{i}=\left[h_{i, 1}, \cdots, h_{i, N_{r}}\right]^{T}$ is the channel vector between the $i$-th transmit antenna and the receiver. The entries of the channel matrix are considered as independent and identicallydistributed (i.i.d.) complex Gaussian random variables with zero mean and unit variance. As in [11], we assume that there is always data available, in the buffer, for transmission. We assume that the CSI is perfectly known at the receiver. To maximize the SNR at the MRC output, the receiver chooses the index of the strongest transmit antenna which is determined by

$$
i_{o}=\arg \max _{1 \leq i \leq N_{t}}\left\|\mathbf{h}_{i}\right\|^{2}=\arg \max _{1 \leq i \leq N_{t}} \sum_{j=1}^{N_{r}}\left|h_{i, j}\right|^{2}
$$

where $\|$.$\| represents the Frobenius norm. We assume that$ the selected index is fed back to the transmitter via an ideal feedback link. The received signal $\mathbf{y} \in \mathbb{C}^{N_{r} \times 1}$ is

$$
\mathbf{y}=\mathbf{h}_{i_{o}} x+\mathbf{n}
$$

where $x$ is the transmitted symbol and $\mathbf{n} \sim \mathcal{C N}\left(0, \sigma_{n}^{2} \mathbf{I}_{N_{r}}\right)$ is the additive complex Gaussian noise.

Furthermore, we assume that over each block duration $T$, the remaining $N_{t}-1$ transmit antennas harvest energy from the environment. The harvested energy is stored in an energy storage device (ESD) [13]. At the end of the frame transmission, the ESD transfers the harvested energy to the transmitter. We note that the harvested energy can be used for the transmission and/or stored for future consumption. Nevertheless, due to the inefficiency of the ESD, an amount of the stored energy can be lost. Hence, the transmitter is encouraged to consume energy as it is harvested [2]. We assume that the transmitter is not connected to any other energy source and draws its power from the ESD. Furthermore, we consider the scenario where all the harvested energy is consumed. We call this scheme a save-and-transmit protocol (S\&T) in contrast with the savethen-transmit (ST) approach presented in [13]. Let $E_{H}$ be the harvested energy over a block duration which is a nonnegative random variable. Therefore, the total power available within a frame duration is $P_{H}=E_{H} / T$. Let $f_{P}(p)$ and $F_{P}(p)$ represent the probability density function (PDF) and the cumulative distribution function (CDF) of $P$, respectively. For the statistical proprieties of the harvested power, we proceed as in [13], where authors have modeled the scavenged power as a gamma random variable. This assumption is justified in [13] by the fact that the Gamma distribution can model many positive random variables. Let $P_{i}$ denotes the power harvested by the $i$-th TX antenna. We assume that the $\left(N_{t}-1\right)$ variables $P_{i}$ are independent and identically distributed (i.i.d.) and follow a gamma distribution with a shape parameter $\alpha_{i}>0$ and a scale parameter $\beta_{i}>0$, i.e.,

$$
P_{i} \sim \operatorname{Gamma}\left(\alpha_{i}, \beta_{i}\right), \quad \text { for } 1 \leq i \leq N_{t} \text { and } i \neq i_{o}
$$

with $\alpha_{1}=\alpha_{2}=\ldots=\alpha_{N_{t}}$ and $\beta_{1}=\beta_{2}=\ldots=\beta_{N_{t}}$. Thus, the total harvested power $P_{H}$ is in turn gamma distributed with shape parameter $\alpha=\left(N_{t}-1\right) \alpha_{i}$ and a scale parameter $\beta=\beta_{i}$, i.e. [17]

$$
P_{H}=\sum_{\substack{i=1 \\ i \neq i_{o}}}^{N_{t}} P_{i} \sim \operatorname{Gamma}\left(\alpha, \beta_{i}\right)
$$

The PDF of the total power $P_{H}$ is given by

$$
f_{P}(p)=\frac{p^{\alpha-1} e^{-p / \beta}}{\beta^{\alpha} \Gamma(\alpha)}
$$

Let $P_{C}$ denotes the circuit power which represents the power consumed by the hardware during the frame transmission and which is assumed constant [13]. The instantaneous received SNR at the MRC output is then

$$
\gamma=\frac{\left\|\mathbf{h}_{i_{o}}\right\|^{2}}{\sigma_{n}^{2}}\left(P_{H}-P_{C}\right)
$$

Given the PDF shown in (5), the average transmitter power is $\bar{P}=\mathbb{E}\left\{P_{H}-P_{C}\right\}=\alpha \beta-P_{C}$. Hence, the average SNR per branch can be defined as

$$
\bar{\gamma}=\left(\alpha \beta-P_{C}\right) / \sigma_{n}^{2}
$$




\section{Outage Probability}

For the MIMO TAS/MRC system, the instantaneous mutual information is [13]

$$
R_{i}=\log _{2}\left(1+\frac{\left\|\mathbf{h}_{i_{o}}\right\|^{2}}{\sigma_{n}^{2}}\left(P_{H}-P_{C}\right)\right)
$$

We note that (8) assumes that $P_{H}>P_{C}$. In fact, if the harvested power is less than the circuit power, there is no transmission and the mutual information is zero. It is also noted that in (8), both $P_{H}$ and $\mathbf{h}_{i_{o}}$ are random variables. For the considered transmission and energy harvesting scenario, the outage event is the union of two mutually exclusive events called the circuit outage and the channel outage [13]. The circuit outage occurs when the available power is less than the circuit power, i.e. $P_{H}<P_{C}$. Clearly, the circuit outage depends on the the statistics of the random variable $P_{H}$. The circuit OP is given by

$$
P_{\text {out }}^{\mathrm{ci}}=\operatorname{Pr}\left\{P_{H}<P_{C}\right\}=F_{P}\left(P_{C}\right)
$$

The channel outage corresponds to the case where $P_{H}>P_{C}$ while the instantaneous mutual information is less than the target rate $R_{T}$. The channel OP depends on the harvested power and on the channel realization $\mathbf{H}$ and is given by

$$
\begin{aligned}
P_{\text {out }}^{\text {ch }} & =\operatorname{Pr}\left\{P_{H}>P_{C}, R_{i}<R_{T}\right\} \\
& =\operatorname{Pr}\left\{P_{H}>P_{C},\left\|\mathbf{h}_{i_{o}}\right\|^{2}<\sigma_{n}^{2} \frac{2^{R_{T}}-1}{P_{H}-P_{C}}\right\} \\
& =\int_{P_{C}}^{+\infty} f_{P}(p) F_{X}\left(\frac{\sigma_{n}^{2}\left(2^{R_{T}}-1\right)}{p-P_{C}}\right) d p
\end{aligned}
$$

where $F_{X}(\cdot)$ is the CDF of $\left\|\mathbf{h}_{i_{o}}\right\|^{2}$, which is given by [18]

$$
F_{X}(x)=\left[1-e^{-x} \sum_{k=0}^{N_{r}-1} \frac{x^{k}}{k !}\right]^{N_{t}}
$$

Since the two outage events are exclusive, the total OP is

$$
\begin{aligned}
P_{\text {out }} & =P_{\text {out }}^{\mathrm{ci}}+P_{\text {out }}^{\mathrm{ch}} \\
& =F_{P}\left(P_{C}\right)+\int_{P_{C}}^{+\infty} f_{P}(p) F_{X}\left(\frac{\sigma_{n}^{2}\left(2^{R_{T}}-1\right)}{p-P_{C}}\right) d p
\end{aligned}
$$

For the general case, it is difficult to obtain a closed-form expression for the integral in (12). Hence, the OP can be evaluated by numerical integration. Nevertheless, when the circuit power is negligible, a closed-form expression can be derived. The following lemma gives an expression for the OP when $P_{C}=0$.

Lemma 1: If the circuit power is negligible, i.e. $P_{C}=0$, the OP is given by

$$
\begin{aligned}
& P_{\text {out }}=1-\frac{2}{\Gamma(\alpha)}\left[\sum_{k=1}^{N_{t}}\left(\begin{array}{c}
N_{t} \\
k
\end{array}\right)(-1)^{k+1} \sum_{l=0}^{\left(N_{r}-1\right) k} a_{k, l} k^{\frac{\alpha-l}{2}} \times\right. \\
& \left.\left(\alpha\left(2^{R_{T}}-1\right)\right)^{\frac{\alpha+l}{2}} \bar{\gamma}^{-\frac{\alpha+l}{2}} K_{\alpha-l}\left(2 \sqrt{\frac{k\left(2^{R_{T}}-1\right) \alpha}{\bar{\gamma}}}\right)\right]
\end{aligned}
$$

where $K_{v}(\cdot)$ is the $v$-th order modified Bessel function of the second kind and $a_{k, l}$ are the coefficient of $x^{l}$ in the expansion $\left[\sum_{i=0}^{N_{r}} \frac{1}{i !} x^{i}\right]^{k}$ defined as: $a_{k, 0}=1, a_{k, 1}=k, a_{k, l}=$ $\frac{1}{l} \sum_{n=1}^{\min \left(l, N_{r}-1\right)} \frac{n(k+1)-l}{n !} a_{k, l-n}$, for $2 \leq l<k\left(N_{r}-1\right)$, and $a_{k, l}=\left[\left(N_{r}-1\right) !\right]^{-k}$, for $l=k\left(N_{r}-1\right)$ [19].

Proof: For $P_{C}=0$, we have $P_{\text {out }}^{\text {ci }}=0$ and the integral giving $P_{\text {out }}^{\text {ch }}$ can be expressed as

$$
\begin{aligned}
P_{\mathrm{out}}^{\mathrm{ch}}= & \int_{0}^{+\infty} \frac{p^{\alpha-1} e^{-p / \beta}}{\beta^{\alpha} \Gamma(\alpha)} F_{X}\left(\frac{\sigma_{n}^{2}\left(2^{R_{T}}-1\right)}{p}\right) d p \\
= & \int_{0}^{+\infty} \frac{p^{\alpha-1} e^{-p / \beta}}{\beta^{\alpha} \Gamma(\alpha)}\left[1-e^{-\sigma_{n}^{2}\left(2^{R_{T}}-1\right) / p}\right. \\
& \left.\times \sum_{k=0}^{N_{r}-1} \frac{\left(\sigma_{n}^{2}\left(2^{R_{T}}-1\right) / p\right)^{k}}{k !}\right]^{N_{t}} d p
\end{aligned}
$$

Thanks to the expansion of $F_{X}(x)$ given in [19], we can write

$$
\begin{aligned}
P_{\text {out }}^{\mathrm{ch}}= & \frac{1}{\beta^{\alpha} \Gamma(\alpha)}\left[\sum_{k=0}^{N_{t}}\left(\begin{array}{c}
N_{t} \\
k
\end{array}\right)(-1)^{k} \times\right. \\
& \left.\quad \sum_{l=0}^{\left(N_{r}-1\right) k} a_{k, l} p_{0}^{l} \int_{0}^{+\infty} p^{\alpha-1-l} e^{-\frac{p}{\beta}-\frac{k p_{0}}{p}} d p\right]
\end{aligned}
$$

Using [20, eq. 2.3.16.1] and after some manipulations, we obtain the result in (13).

\section{ERgodic Channel CAPACITY}

In this section, we derive a closed-form expression of the ergodic capacity when the circuit power is negligible. The ergodic channel capacity can be expressed as

$C=\int_{x=0}^{+\infty} \int_{p=P_{C}}^{+\infty} \log _{2}\left(1+\frac{x\left(p-P_{C}\right)}{\sigma_{n}^{2}}\right) f_{X}(x) f_{P}(p) d x d p$

where $f_{X}(x)$ is the PDF of $\left\|\mathbf{h}_{i_{o}}\right\|^{2}$ given by

$$
f_{X}(x)=\frac{N_{t}}{\left(N_{r}-1\right) !} x^{N_{r}-1} e^{-x}\left[1-e^{-x} \sum_{k=0}^{N_{r}-1} \frac{x^{k}}{k !}\right]^{N_{t}-1}
$$

Let us assume that $P_{C}=0$. Alternatively, the channel capacity can be written as

$$
C=\int_{z=0}^{+\infty} \log _{2}(1+\gamma) f_{\Gamma}(\gamma) d \gamma
$$

where $f_{\Gamma}(\gamma)$ is the PDF of $\gamma=\frac{\left\|\mathbf{h}_{i_{o}}\right\|^{2}}{\sigma_{n}^{2}} P_{H}$ given by

$$
\begin{aligned}
f_{\Gamma}(\gamma)= & \int_{0}^{+\infty} f_{\Gamma \mid P}(\gamma \mid p) f_{P}(p) d p \\
= & \int_{0}^{+\infty} \frac{\sigma_{n}^{2}}{p} f_{X}\left(\frac{\sigma_{n}^{2}}{p} \gamma\right) f_{P}(p) d p \\
= & \frac{2 N_{t}}{\left(N_{r}-1\right) ! \Gamma(\alpha)} \sum_{k=0}^{N_{t}-1}\left(\begin{array}{c}
N_{t}-1 \\
k
\end{array}\right)(-1)^{k} \\
& \times\left[\sum_{l=0}^{\left(N_{r}-1\right) k} a_{k, l}\left(\frac{\alpha}{\bar{\gamma}}\right) \frac{\left(\alpha+l+N_{r}\right)}{2}(k+1)^{\left(\alpha-l-N_{r}\right) / 2}\right. \\
& \left.\times \gamma^{\frac{\left(\alpha+l+N_{r}\right)}{2}-1} k_{\alpha-l-N_{r}}\left(2 \sqrt{\frac{(k+1) \alpha \gamma}{\bar{\gamma}}}\right)\right]
\end{aligned}
$$


Substituting (19) into (18) and using the relationship in [21, eq. 13], we can express the ergodic capacity as

$$
\begin{aligned}
& C=\int_{z=0}^{+\infty} \log _{2}(1+\gamma) f_{\Gamma}(\gamma) d \gamma \\
& =\frac{2 N_{t}}{\left(N_{r}-1\right) ! \Gamma(\alpha) \ln (2)} \sum_{k=0}^{N_{t}-1}\left(\begin{array}{c}
N_{t}-1 \\
k
\end{array}\right)(-1)^{k} \\
& \times \sum_{l=0}^{\left(N_{r}-1\right) k} a_{k, l}(k+1)^{\frac{\alpha-l-N_{r}}{2}}\left(\frac{\alpha}{\bar{\gamma}}\right)^{\frac{\alpha+l+N r}{2}}
\end{aligned}
$$

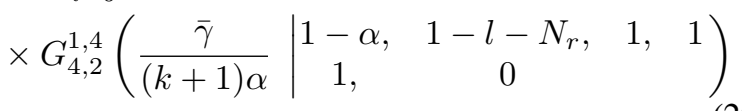

where $G_{p, q}^{m, n}\left(\cdot \mid \begin{array}{l}a_{p} \\ b_{q}\end{array}\right)$ is the Meijer-G function defined in [22].

\section{Average Symbol ERror Rate}

It is well known that the instantaneous SER of many digital modulations can be expressed as [23]

$$
P_{e}(\gamma)=\frac{c}{2} \operatorname{erfc}(\sqrt{b \gamma})
$$

where $c$ and $b$ are modulation specific constants and $\operatorname{erfc}(\cdot)$ is the the complementary error function which can be expressed in terms of the Meijer $G$-function as [22, eq. 8.4.14.2]

$$
\operatorname{erfc}(\sqrt{x})=\frac{1}{\sqrt{\pi}} G_{1,2}^{2,0}\left(x \mid \begin{array}{ll}
1, & \\
0, & \frac{1}{2}
\end{array}\right)
$$

The average SER $\bar{P}_{e}$ can be derived by averaging the instantaneous SER over instantaneous SNRs, i.e.,

$$
\bar{P}_{e}=\mathbb{E}\left\{P_{e}(\gamma)\right\}=\int_{0}^{\infty} P_{e}(\gamma) f_{\Gamma}(\gamma) d \gamma
$$

By substituting (19) and (22) into (23) and calculating the resultant integral with the aid of [24, eq. (7.821.3)], we obtain a closed-form SER expression as

$$
\begin{aligned}
& \bar{P}_{e}=\frac{N_{t} c}{\left(N_{r}-1\right) ! \Gamma(\alpha)} \sum_{k=0}^{N_{t}-1}\left(\begin{array}{c}
N_{t}-1 \\
k
\end{array}\right)(-1)^{k} \\
& \times\left[\sum_{l=0}^{\left(N_{r}-1\right) k} a_{k, l}(k+1)^{-l-N_{r}}\right.
\end{aligned}
$$

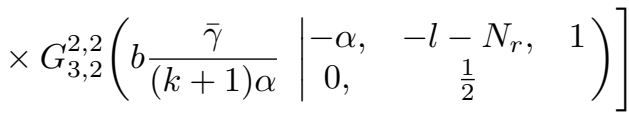

\section{NuMERicAl RESUlts}

This section presents some numerical results that illustrate our theoretical analysis for different MIMO configurations. We assume that the target rate is $R_{T}=2 \mathrm{bits} / \mathrm{s} / \mathrm{Hz}$.

In Fig. 1, we compare the OP for different MIMO configurations, $P_{C}=0$, and a fixed mean power $\bar{P}=\alpha \beta=50$ $\mathrm{J} / \mathrm{s}$. In our simulations, the SNR given by (7) is obtained by varying the noise variance $\sigma_{n}^{2}$ while keeping the average harvested energy constant. The analytical curves are obtained based on expression (13) in Lemma 1. Fig. 1 indicates an excellent match between the exact analytical OP and the MC

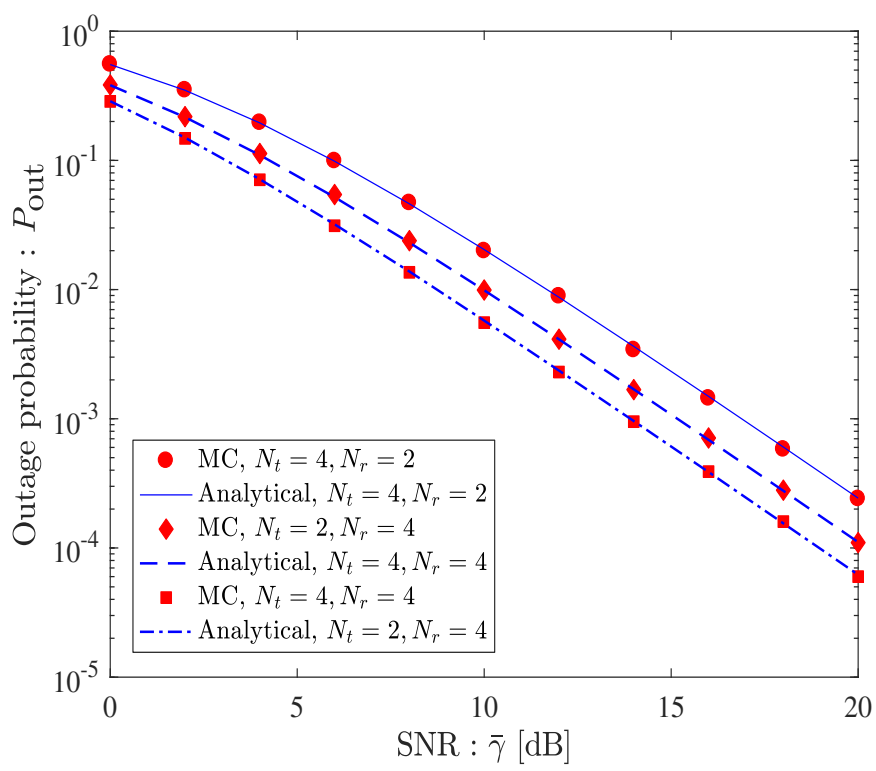

Fig. 1. OP versus SNR for $P_{C}=0$, mean power $\bar{P}=\alpha \beta=50 \mathrm{~J} / \mathrm{s}$ and different MIMO configurations.

simulated curves. We note that as the number of used antenna increases, the OP decreases. However, we can see that the $(2 \times 4)$-MIMO system outperforms the $(4 \times 2)$-MIMO one.

Fig. 2 shows the impact of the gamma distribution parameter $\beta$ on the OP. Similarly to Fig. 1, the analytical curves are obtained based on expression (13). The curves are obtained by fixing the mean power to $\bar{P}=50 \mathrm{~J} / \mathrm{s}$ and varying $\beta$ for the $(4 \times 4)$-MIMO system. For further comparison, the OP for system with constant deterministic power $\bar{P}$ (without $\mathrm{EH}$ ) is also presented. As shown, an OP loss is noted for systems with random power. However, when $\beta$ decreases, the OP of the EH TX system approaches that of the constant power scheme. We note that $\beta$, the scale parameter of the gamma distribution, determines the statistical dispersion of the harvested random energy. When $\beta$ is large, the distribution of the random harvested energy will be more spread out. However, when $\beta$ decreases, the distribution will be more concentrated and the harvested energy exhibits less randomness.

In Fig. 3, the OP as a function of the number of transmit antennas $N_{t}$ is plotted for different values of $\sigma_{n}^{2}$. Curves are obtained by varying the $N_{t}$ parameter in (13). We can see that increasing the number of transmit antennas will improve the OP since the amount of the harvested energy increases. Fig. 4 illustrates the effect of the circuit power on the OP for the $(4 \times$ 4)-MIMO system and different values of $P_{C}$. The analytical curves are generated using (12), where the integral is evaluated numerically. As expected, the figure shows perfect agreement between the numerical analysis and the MC simulations. We can see that the OP increases dramatically with the circuit power.

In Fig. 5, we compare the channel capacity for different values of $\beta$. This figure gives also the channel capacity for the MIMO system with constant power. It is shown that the simulation curves match well with the theoretical result given by (20). We can see that as $\beta$ decreases, the channel capacity 


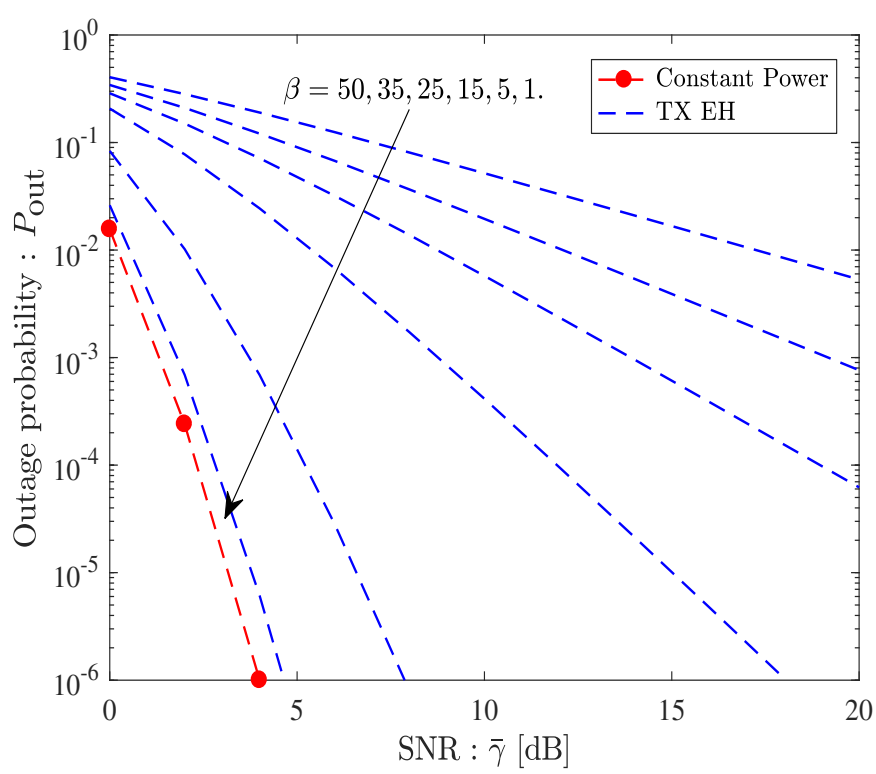

Fig. 2. Impact of $\beta$ on the OP for the $(4 \times 4)$-MIMO system.

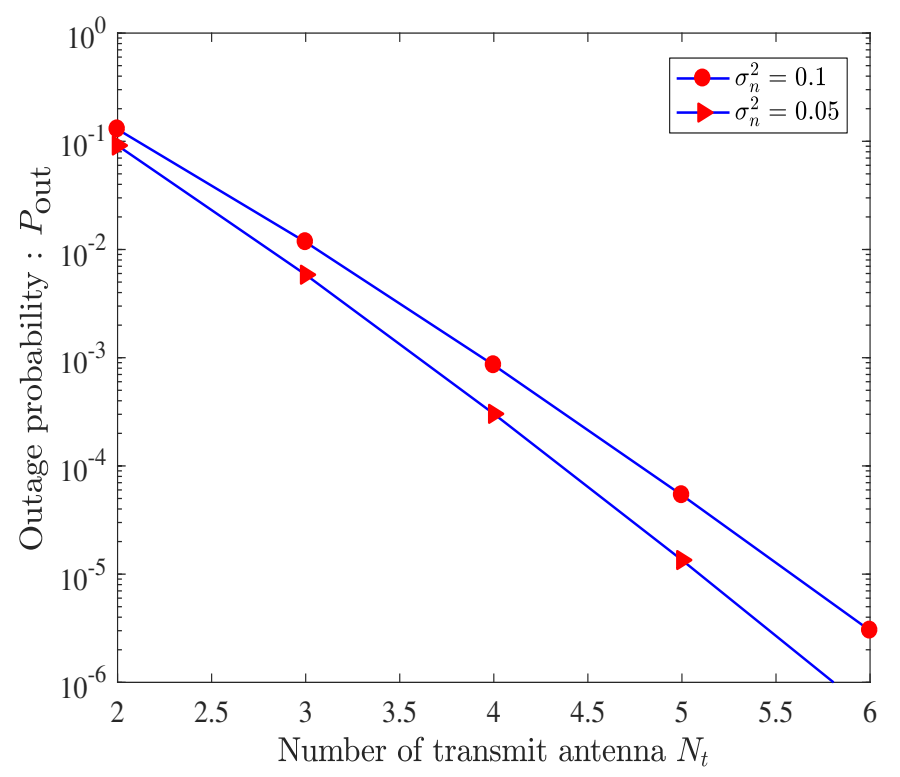

Fig. 3. The OP versus $N_{t}$ for $N_{r}=4, \alpha_{i}=0.5$, and $\beta=5$.

curve approaches the scenario with deterministic constant power. For $\beta=50$, the capacity loss due to the energy randomness is about $1 \mathrm{bit} / \mathrm{s} / \mathrm{Hz}$.

Fig. 6 presents the performance comparison between the SER expression (24) and the MC simulation results. The results are obtained for quadrature phase-shift keying (QPSK) modulation $(a=2, b=0.5)$. The average SER of the MIMO TAS/MRC system without EH is also presented. We can see that there is an excellent agreement between the analytical SER expression and MC results. Once again, when the scale parameter $\beta$ decreases, the SER curves for the S\&T protocol approach the case of the system with deterministic power.

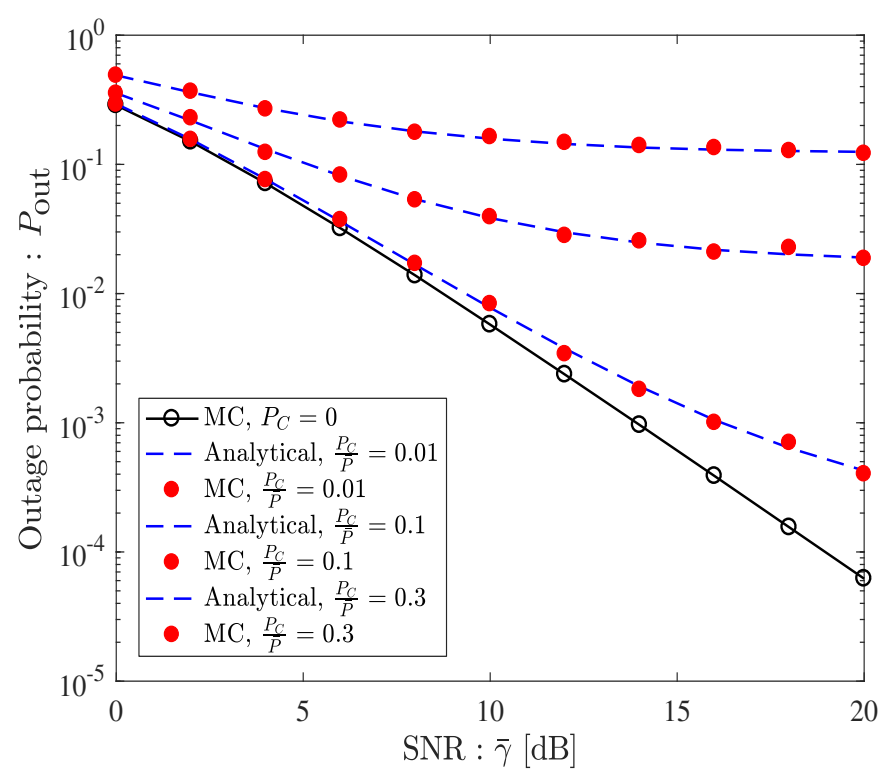

Fig. 4. Impact of the circuit power $P_{C}$ on the OP for the $(4 \times 4)$-MIMO system.

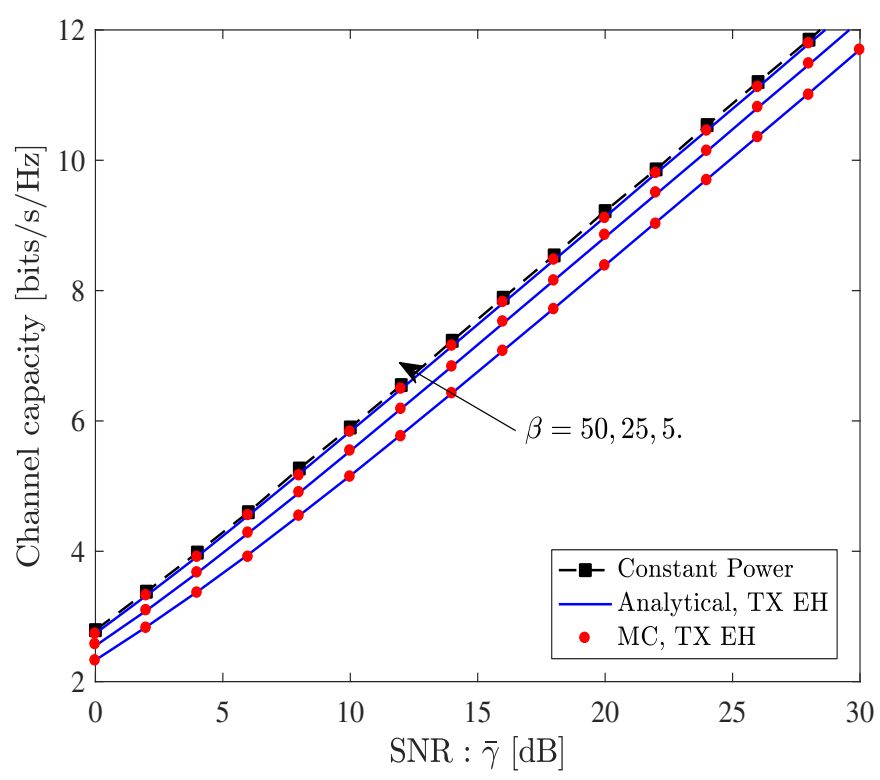

Fig. 5. Channel capacity for the $(4 \times 4)$-MIMO system, $P_{C}=0$, and different values of $\beta$.

\section{CONCLUSION}

In this paper, we proposed a wireless MIMO system where the TX relies completely on EH. We assume that the receiver is based on the TAS/MRC techniques. Hence, only a single antenna is used for the data transmission at the TX. So, we propose to use the remaining transmit antennas to harvest energy form natural sources. This S\&T protocol allows the data transfer with the TX powered exclusively by EH as for the ST technique proposed in [13] for SISO systems. Furthermore, an analytical performance assessment of the proposed protocol over Rayleigh flat fading channels was presented. Expressions for the CDF and PDF of the SNR at the MRC combiner output 


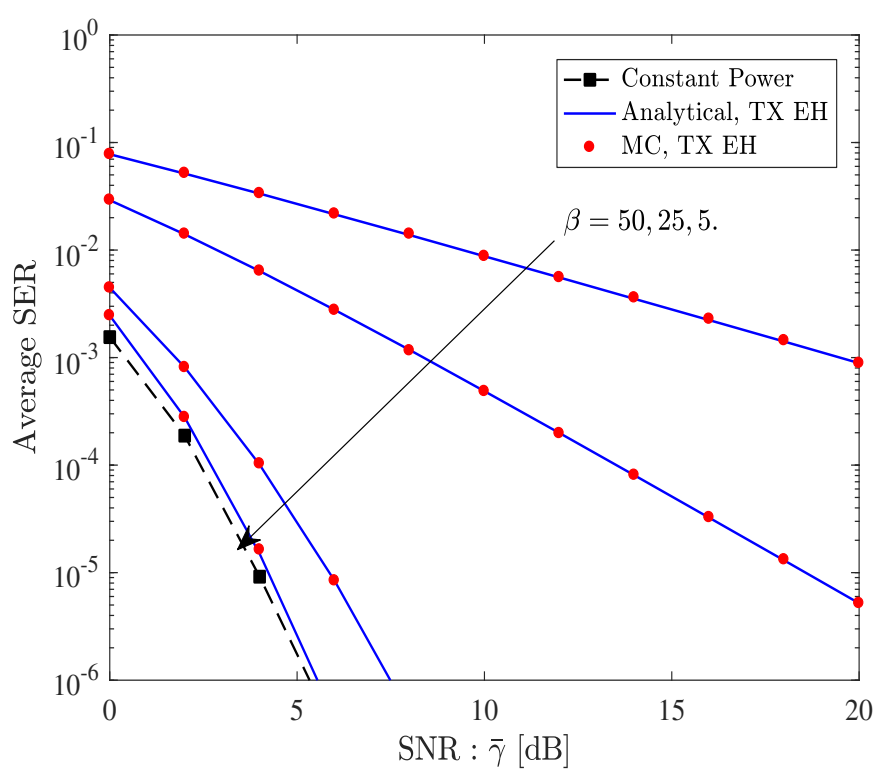

Fig. 6. SER of QPSK for $(4 \times 4)$-MIMO system, $P_{C}=0$, and different values of $\beta$.

were derived. Then, we have derived the expressions for the OP, the average SER and the channel capacity. Numerical results have shown an excellent agreement between analytical expressions and MC simulation curves. In our analysis, we have assumed that the random harvested power is gamma distributed. We noted that when the scale parameter of the gamma distribution is small, the performances of the MIMO EH TX scheme approach those of systems with constant deterministic power. In fact, for small values of the scale parameter, the distribution is concentrated and this corresponds to less randomness in the amount of harvested energy.

The main difference between our protocol and the ST technique presented in [13] is that the former is based on the time switching approach, while our protocol is based on antenna selection. With the time switching method, the frame interval is fractionated in two intervals. A fraction is used exclusively for $\mathrm{EH}$ and the other for the data transmission. Our protocol allows simultaneous energy harvesting and data transmission. The proposed autonomous transmitter can represent an efficient solution for some particular cases, especially when the available power amount is insufficient. However, its practical implementation needs more investigation that was unaddressed in this paper. More specific statistical models for modeling the harvested power should be studied. Moreover, the circuit power should be considered in the performance analysis. It would also be interesting to adapt the transmission rate based on the CSI and the ESI. If the amount of the harvested energy exceeds a certain threshold, it will be preferable to store a part of this energy for future use. The optimization of a such approach is worth further exploration.

\section{REFERENCES}

[1] O. Ozel, K. Tutuncuoglu, J. Yang, S. Ulukus, and A. Yener, "Transmission with Energy Harvesting Nodes in Fading Wireless Channels: Optimal Policies," IEEE J. Sel. Areas Commun., vol. 29, no. 8, pp. 1732-1743, Sep. 2011, DOI: 10.1109/JSAC.2011.110921.
[2] K. Tutuncuoglu and A. Yener, "Optimum Transmission Policies for Battery Limited Energy Harvesting Nodes," IEEE Trans. Wireless Commun., vol. 11, no. 3, pp. 1180-1189, Mar. 2012, DOI: 10.1109/TWC.2012.012412.110805.

[3] J. Yang and S. Ulukus, "Optimal Packet Scheduling in an Energy Harvesting Communication System," IEEE Trans. Commun., vol. 60, no. 1, pp. 220-230, Jan. 2012, DOI: 10.1109/TCOMM.2011.112811.100349.

[4] O. Ozel and S. Ulukus, "Achieving AWGN Capacity Under Stochastic Energy Harvesting," IEEE Trans. Inf. Theory, vol. 58, no. 10, pp. 64716483, Oct. 2012, DOI: 10.1109/TIT.2012.2204389.

[5] B. Devillers and D. Gunduz, "A General Framework for the Optimization of Energy Harvesting Communication Systems with Battery Imperfections," J Commun Networks, vol. 14, no. 2, pp. 130-139, Apr. 2012, DOI: 10.1109/JCN.2012.6253061.

[6] D. Gunduz, K. Stamatiou, N. Michelusi, and M. Zorzi, "Designing Intelligent Energy Harvesting Communication Systems," IEEE Commun. Mag., vol. 52, no. 1, pp. 210-216, Jan. 2014, DOI: 10.1109/MCOM.2014.6710085.

[7] M. L. Ku, W. Li, Y. Chen, and K. J. R. Liu, "Advances in Energy Harvesting Communications: Past, Present, and Future Challenges," IEEE Communications Surveys Tutorials, vol. 18, no. 2, pp. 1384-1412, Sep. 2016, DOI: 10.1109/COMST.2015.2497324.

[8] C. K. Ho and R. Zhang, "Optimal Energy Allocation for Wireless Communications With Energy Harvesting Constraints," IEEE Trans. Signal Process., vol. 60, no. 9, pp. 4808-4818, Sep. 2012, DOI: 10.1109/TSP.2012.2199984.

[9] V. Sharma, U. Mukherji, V. Joseph, and S. Gupta, "Optimal energy management policies for energy harvesting sensor nodes," IEEE Trans. Wireless Commun., vol. 9, no. 4, pp. 1326-1336, Apr. 2010, DOI: 10.1109/TWC.2010.04.080749.

[10] Z. Wang, V. Aggarwal, and X. Wang, "Power Allocation for Energy Harvesting Transmitter With Causal Information," IEEE Trans. Commun., vol. 62, no. 11, pp. 4080-4093, Nov 2014, DOI: 10.1109/TCOMM.2014.2357430.

[11] M. R. Zenaidi, Z. Rezki, and M. S. Alouini, "Performance Limits of Online Energy Harvesting Communications With Noisy Channel State Information at the Transmitter," IEEE Access, vol. 5, pp. 1239-1249, 2017, DOI: 10.1109/ACCESS.2017.2654454.

[12] J. Gómez-Vilardebó, "Competitive Design of Energy Harvesting Communications in Wireless Fading Channels," IEEE/ACM Trans. Netw., vol. 25, no. 6, pp. 3863-3872, Dec. 2017, DOI: 10.1109/TNET.2017.2757515.

[13] S. Luo, R. Zhang, and T. J. Lim, "Optimal Save-Then-Transmit Protocol for Energy Harvesting Wireless Transmitters," IEEE Trans. Wireless Commun., vol. 12, no. 3, pp. 1196-1207, Mar. 2013, DOI: 10.1109/TWC.2013.012413.120488.

[14] S. Shirvani Moghaddam, "Outage Analysis of Energy Harvested RelayAided Device-to-Device Communications in Nakagami Channel," Journal of Communications Software and Systems, vol. 14, no. 4, pp. 302 311, Dec. 2018, DOI: 10.24138/jcomss.v14i4.490.

[15] L. Pantoli, A. Leoni, V. Stornelli, and G. Ferri, "An IC architecture for RF Energy Harvesting systems," Journal of Communications Software and Systems, vol. 13, no. 2, pp. 96-100, Jun. 2017, DOI: 10.24138/jcomss.v13i2.377.

[16] S. Thoen, L. Van der Perre, B. Gyselinckx, and M. Engels, "Performance Analysis of Combined Transmit-SC/Receive-MRC," IEEE Trans. Commun., vol. 49, no. 1, pp. 5-8, 2001, DOI: 10.1109/26.898241.

[17] S. Al-Ahmadi and H. Yanikomeroglu, "On the Approximation of the Generalized- $K$ Distribution by a Gamma Distribution for Modeling composite fading Channels," IEEE Trans. Wireless Commun., vol. 9, no. 2, pp. 706-713, feb 2010, DOI: 10.1109/TWC.2010.02.081266.

[18] Z. Chen, J. Yuan, and B. Vucetic, "Analysis of Transmit Antenna Selection/Maximal-Ratio Combining in Rayleigh Fading Channels,' IEEE Trans. Veh. Technol., vol. 54, no. 4, pp. 1312-1321, 2005, DOI: 10.1109/TVT.2005.851319

[19] C. J. Chen and L.-C. Wang, "A Unified Capacity Analysis for Wireless Systems with Joint Multiuser Scheduling and Antenna Diversity in Nakagami Fading Channels," IEEE Trans. Commun., vol. 54, no. 3, pp. 469-478, Mar. 2006, DOI: 10.1109/TCOMM.2005.863778.

[20] A. P. Prudnikov, Y. A. Brychkov, and O. I. Marichev, Integrals \& Series V1: Elementary Functions. CRC, 1998.

[21] A. Muller and J. Speidel, "Capacity of Multiple-Input Multiple-Output Keyhole Channels with Antenna Selection," in in Proc. Eur. Wireless Conf., Paris, France, 2007.

[22] A. P. Prudnikov, Y. A. Brychkov, and O. I. Marichev, Integrals \& Series V3: More Special Functions. CRC, 1990. 
[23] J. Proakis and M. Salehi, Digital Communications, 5th ed. McGrawHill Higher Education, 2007.

[24] I. Gradshteyn and I. Ryzhik, Table of Integrals, Series, and Products, 7th ed. Academic Press, 32007.

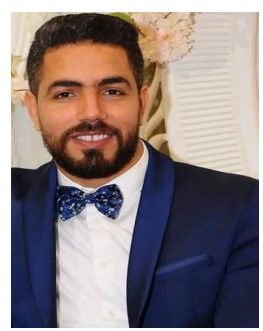

Issa Chihaoui is currently a Ph.D student at National Engineering School of Tunis. He received the Engineering and the M.Sc. degrees from the University of Sousse, Sousse, Tunisia, in 2009 and 2012, respectively. He is an active member in the Innov'COM Laboratory, Sup'Com, University of Carthage. His research interests include channel equalization, massive MIMO, signal detection and cooperative systems.

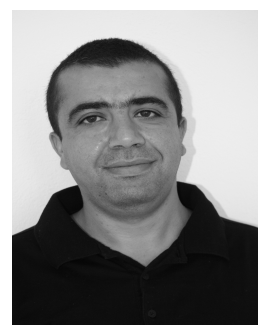

Mohamed Lassaad Ammari received the Engineering degree from the University of Carthage, Tunis, Tunisia, in 1995 and the M.Sc. and the Ph.D. Degrees from Université Laval, Quebec City, QC, Canada, in 2000 and 2003, respectively. From 2003 to 2005 , he was a research associate with the Laboratory of Communications and Integrated Microelectronics, École de Technologie Supérieure, Montreal, QC. He is currently a Professor with the National Engineering School of Sousse, Tunisia. He is also an active team member with the NOCCS Laboratory, University of Sousse). His research interests include channel equalization, multiple-inputmultiple-output orthogonal frequency-division multiplexing systems, turbo detection, space-time coding, and adaptive modulation. 